TITLE:

\title{
Pore structure of aluminas derived from the alkyl derivatives of boehmite
}

$\operatorname{AUTHOR}(S)$ :

Kim, Sung-Wook; Iwamoto, Shinji; Inoue, Masashi

CITATION:

Kim, Sung-Wook ...[et al]. Pore structure of aluminas derived from the alkyl derivatives of boehmite. Journal of Porous Materials 2009, 16(5): 605-612

ISSUE DATE:

2009-10

URL:

http://hdl.handle.net/2433/87160

\section{RIGHT:}

c 2008 Springer Science+Business Media, LLC.; この論文は出版社版で ありません。引用の際には出版社版をご確認ご利用ください。; This is not the published version. Please cite only the published version. 


\title{
Pore structure of aluminas derived from the alkyl derivatives of boehmite
}

\author{
Sung-Wook Kim, Shinji Iwamoto, Masashi Inoue*
}

Department of Energy and Hydrocarbon Chemistry, Graduate School of Engineering,

Kyoto University, Katsura, Kyoto 615-8510, Japan

Corresponding author. Tel.: +81-75-383-2478; fax: 81-75-383-2479.

E-mail address: inoue@scl.kyoto-u.ac.jp(M. Inoue) 


\section{Abstract}

2 The alkyl derivatives of boehmite (alkoxyalumoxanes; $\mathrm{AlO}(\mathrm{OH})_{1-\mathrm{x}}(\mathrm{OR})_{\mathrm{x}}$ ) were

3 synthesized by the reaction of aluminum triisopropoxide in straight-chain primary

4 alcohols at $300{ }^{\circ} \mathrm{C}$ for $2 \mathrm{~h}$ in an autoclave. In the present work, pore structures of

5 aluminas obtained by calcination of the alkyl derivative of boehmite were examined.

6 The alumina obtained from the ethyl derivative of boehmite had a broad pore-size

7 distribution, while the pore-size of the alumina obtained from the dodecyl derivative of

8 boehmite distributed in a narrow range in the mesopore region. The mode pore

9 diameter of the latter alumina increased with the increase in calcination temperature

10 (as-syn., $39 \AA \AA$; $600{ }^{\circ} \mathrm{C}, 54 \AA$; $800{ }^{\circ} \mathrm{C}, 58 \AA$; 1000 $\mathrm{C}, 68 \AA$ ), but narrow pore-size

11 distribution was maintained even after calcination at high temperatures.

Keywords: Alumina; Pore structure; Solvothermal reaction 


\section{Introduction}

20 For the supports of industrial catalysts, alumina is most widely used because it is

21 inexpensive and reasonably stable, and is provided with a wide range of surface areas and porosities suitable for a variety of catalyst application [1]. Preparation of alumina

23 (or alumina-based) supports with the controlled pore structure is still active area: Thus, many papers have been reported [2-8].

Boehmite is one of the modifications of aluminum oxide hydroxide, $\mathrm{AlOOH}$, and it can be easily prepared by hydrothermal treatment of aluminum hydroxide [9]. Microcrystalline boehmite is called "pseudoboehmite" and is used as a precursor of aluminas. Although boehmite has a layer structure, intercalation of guest molecules into the boehmite layers has never been reported, presumably because of strong hydrogen bonding between the layers. On the other hand, during the course of our long-term study on controlling the pore texture of alumina for use as catalyst supports, we found that the thermal treatment (glycothermal and alcohothermal treatments) of aluminum triisopropoxide (AIP) in organic solvents (glycols and alcohols) yielded novel derivatives of boehmite, in which the alkyl (or hydroxyalkyl) groups were incorporated into the boehmite layers through the covalent bondings [10-13]. 
derivatives of boehmite were examined.

\section{Experimental}

\subsection{Synthesis of the alkyl derivatives of boehmite}

In a Pyrex test tube serving as an autoclave liner, $130 \mathrm{ml}$ of a straight-chain primary

alcohol (ethanol, EtOH; 1-butanol, BuOH; 1-pentanol, PeOH; 1-hexanol, $\mathrm{HeOH}$;

1-octanol, OcOH; 1-decanol, DeOH; 1-dodecanol, DDOH) and 12.5 g of aluminum

triisopropoxide (AIP) were placed, and the test tube was then placed in a $300 \mathrm{ml}$ autoclave. In the gap between the autoclave wall and the test tube was placed an

additional $30 \mathrm{ml}$ of the alcohol. The autoclave was thoroughly purged with nitrogen,

heated to $300{ }^{\circ} \mathrm{C}$ at a rate of $2.3^{\circ} \mathrm{C} / \mathrm{min}$, and held at that temperature for $2 \mathrm{~h}$. After the mixture was cooled to room temperature, the resulting precipitate was washed by repeated cycles of agitation with methanol, centrifuging, and decantation, and then air-dried. The obtained product was calcined at the various temperatures by heating at a rate of $10^{\circ} \mathrm{C} / \mathrm{min}$ and holding at that temperature for $30 \mathrm{~min}$ in a furnace in static air.

52 These products will be designated by " $\mathrm{A}$ " followed by the abbreviation for the medium used in the alcohothermal treatment and calcination temperature in degree 
54 Celsius in parentheses. The original samples will be specified by a term, “as-syn”, in

55

56

57

58

59

60

61

62

63

64 parentheses.

\subsection{Characterization.}

Powder X-ray diffraction (XRD) was measured on a Shimadzu XD-D1 diffractometer using $\mathrm{CuK} \alpha$ radiation and a carbon monochromator. The nitrogen adsorption isotherms were measured at liquid-nitrogen temperature by using a volumetric gas-sorption system, Quantachrome Autosorb-1. The alumina samples were previously outgassed at $300{ }^{\circ} \mathrm{C}$ for $30 \mathrm{~min}$. Surface areas were calculated by applying the BET method to the adsorption data, taking the average area occupied by a nitrogen molecule as $0.162 \mathrm{~nm}^{2}$. Pore size distributions were calculated from the desorption branch of the nitrogen adsorption isotherm by the BJH method. Infrared spectra were obtained on JASCO FT/IR-470 plus spectrometer using the usual KBr-pellet technique with 128 integration times. Simultaneous thermogravimetric (TG) and differential thermal analyses (DTA) were performed on a Shimadzu DTG-50 analyzer: a weighed amount (ca. $20 \mathrm{mg}$ ) of the sample was placed in the analyzer, and then heated at the rate of $10{ }^{\circ} \mathrm{C} / \mathrm{min}$. Morphologies of the products were observed with a scanning electron microscope (SEM), Hitachi S-2500X. 


\section{Results and discussion}

\subsection{Alkyl derivatives of boehmite obtained by solvothermal method.}

The XRD patterns of the products are shown in Fig. 1. For comparison, the XRD pattern of pseudoboehmite is also given in the figure. The XRD peaks at the high angle side $\left(2 \theta=50^{\circ}\right.$ and $\left.65^{\circ}\right)$ correspond to the lattice parameters, $a$ and $c$, of pseudoboehmite (200 and 002 planes), and the obtained products could be indexed on the basis of the boehmite structure [12,13]. The 020 plane of products shifted toward the lower-angle side with the increase in the carbon number of the alcohol used as the solvent (Fig. 1), suggesting that the alkyl groups derived from the solvent alcohol are incorporated between the boehmite layers. In the XRD patterns of $\mathrm{ADeOH}(\mathrm{as}-\mathrm{syn})$ and ADDOH(as-syn), the peak due to 020 plane was not clearly shown. The alkyl chains of the decyl and dodecyl groups are so long, that the diffraction peaks for the 020 plane (basal plane) of ADeOH and ADDOH appeared at $2 \theta<3^{\circ}$. However, they exhibited the 040 and 060 diffraction peaks (these peaks are 2nd and 3rd order diffraction peaks of the basal plane), and therefore the position of the 020 diffraction peak can be calculated precisely from these peaks, which clearly shows the low-angle shift of the 020 diffraction peak. To clarify these points, the 040 and 060 diffraction peaks are indexed in Fig. 1. Linear increase of basal spacing with the increase of carbon number 
90 of solvents was observed (Supplementary materials, Fig. 1S), which is an incontestable

91 evidence for the incorporation of the alkyl groups derived from the solvent alcohols.

92 In the IR spectra of the products shown in Fig. 2, bands characteristic of the

93 boehmite layers are seen at around 615 and $480 \mathrm{~cm}^{-1}$ [14-16], suggesting that the

94 products had the layer structure of boehmite. Bands due to the incorporated organic moieties were also noted at $3000-2850 \mathrm{~cm}^{-1}\left(v_{\mathrm{CH}}\right)$.

Figure 3 shows the results for thermal analyses of the products. A large weight decrease is seen around 100 and $400{ }^{\circ} \mathrm{C}$ for all of the samples. In the DTA profiles of the products, one endothermic and two exothermic processes took place at around 100, 300 and $400{ }^{\circ} \mathrm{C}$, respectively. The first process is attributed to desorption of 100 physisorbed water/methanol and the second and third processes are caused by combustion of the alkyl moieties incorporated between the boehmite layers. Collapse

102 of the boehmite layers yielding amorphous alumina seems to take place simultaneously with the last process because of large exothermic effects. Because the molecular

104 weight of ethanol is small as compared to the other alcohols used in this study, the intensity of the exothermic peak as well as the total weight decrease is the smallest among the samples. The exothermic peak shifted slightly toward lower temperature 
$108 \mathrm{ADDOH}\left(\right.$ as-syn); $373{ }^{\circ} \mathrm{C}$ ). This result may be attributed to the fact that the octane

109 number of an alcohol having a shorter alkyl chain is higher than that of alcohol having

110 a longer alkyl chain [17]. The results of thermal analyses of the products obtained by

111 the reaction of AIP in primary alcohols are summarized in Table 1. All these results are

112 consistent with the fact that the alkyl moieties are incorporated between the boehmite

113 layers.

114

115

3.2. Morphological aspects.

116 The scanning electron micrographs of the products are shown in Fig. 4. Particles of

117 the product obtained in ethanol (AEtOH(as-syn)) had a rod shape. On the other hand,

118 particles of APeOH(as-syn) had irregular shapes but rod shape particles were also seen

119 together with the irregularly-shaped particles. AHeOH(as-syn) and ADDOH(as-syn)

120 were composed of large aggregate particles having irregular shapes.

\subsection{Calcined products.}

Phase transformation of the alkyl derivatives of boehmite was investigated and Fig.

5 shows the transformation of $\mathrm{AHeOH}$ as the representative results. $\mathrm{AHeOH}$ maintained the boehmite structure at $300{ }^{\circ} \mathrm{C}$ and converted to amorphous alumina at 
$126400{ }^{\circ} \mathrm{C}$ (Fig. 5). The $\gamma$-phase appeared by calcination in air at $800{ }^{\circ} \mathrm{C}$ for $30 \mathrm{~min}$. The

127 sample calcined at $1000{ }^{\circ} \mathrm{C}$ was composed of $\theta$ - and $\alpha$-phases. The single-phase of

$128 \alpha$-phase was obtained by calcination at $1200{ }^{\circ} \mathrm{C}$.

129 All of the other alkyl derivatives of boehmite were converted into $\gamma$-alumina

130 through an amorphous phase by calcination at $1000{ }^{\circ} \mathrm{C}$ in air, and the XRD patterns

131 were essentially identical irrespective of the solvents used.

132 Nitrogen adsorption isotherms, and pore size distribution curves of some the

133 products are shown in Figs. 6-8 and t-plots of all the products examined in this paper

134 are given in Fig. 9.

135 Although the aluminas derived from the ethyl derivative of boehmite exhibited rather

136 complex $\mathrm{N}_{2}$-adsorption isotherms (Fig. 6a), the $t$-plots (Fig. 9a) derived from the

137 isotherms can be divided into 5 segments. At low $t$ (statistical thickness; 0-3 Å) region

138 (corresponding to low partial pressure of nitrogen), the plot seems to have a rather steep slope going through the origin, although only a few data points are available. In

140 the second segment (3-7 $\AA$ ), the slope decreased. The slope increased in the third segment (7-12 $\AA)$ and decreased again in the forth segment (12-18 $\AA$ ) with final increase at higher $t$ region (>18 $\AA$; fifth segment). The decrease of the slope from the

143 first to second segment is a typical phenomenon for microporous materials and is 
144 explained by micropore filling. It was reported that calcination of well-crystallized

145 boehmite gives alumina having micropores with a slit-shape $[18,19]$. The crystallite

146 sizes of the present products were relatively large, and much larger than

147 pseudoboehmite usually obtained in aqueous systems. Therefore, the boehmite layer

148 structure seems to give the slit-shaped micropores on calcination. Hysteresis loop

149 observed in the isotherms (Fig. 6a) supports the presence of slit-shaped pores in the

$150 \mathrm{AEtOH}(600)$.

151 The increase in the slope at the middle partial pressure region (third segment) can be

152 explained by capillary condensation of adsorbate molecules into mesopores. Gradual

153 decrease in the slope in the third and forth segments suggests that the pore size

154 distributed widely, which is verified by the pore-size distribution curve calculated by

155 the BJH method (Fig. 6b). These pores seem to be formed in the rod-shaped

156 pseudomorphous particles because of the difference between the true densities of

$157 \gamma$-alumina and the ethyl derivative of boehmite. The large slope in the fifth segment

158 indicates the presence of macropores, which can be also recognized from the pore-size

159 distribution curve shown in Fig. 6b. Since the pore size is in the order of the size of

160 rod-shaped particles, these pores are formed between pseudomorphous rod-shaped particles. 
alkyl groups during the drying stage prior to the $\mathrm{N}_{2}$-adsorption measurement.

164 Calcination of the product developed micro- and meso-pores because of elimination of ethyl groups and collapse of the boehmite layer structure. Significant increase in mesopore volume is apparent. Further increase in the calcination temperature caused a significant decrease in micropore volume while mesopore volume was slightly decreased. This result suggests that the primary particles of alumina separated by micropores are easily sintered by heat treatment. On the other hand, macropores

170 formed between the rod-shaped particles was not affected by calcination. This can be recognized by essentially identical slopes in the fifth segment and also by the pore-size distribution curves.

173 The aluminas derived from $\mathrm{ABuOH}$ showed $t$-plots (Fig. 9b) different from those obtained from AEtOH. The decrease of the slope from the first to second segments was not seen, indicating that the micropores were not present in the samples. The increase in the slope from the second to third segment was significant and third and forth segments are clearly distinguished, indicating that the pore size distributed in a narrow range, which is verified by the pore-size distribution curve (Fig. 7b). Macropores were not recognized. Hysteresis loop of these products suggests that tubular pores with 
narrow constriction were formed (Fig. 7a).

181 Nitrogen adsorption isotherms of $\mathrm{AOcOH}$ exhibited a similar tendency with $\mathrm{ABuOH}$.

182 However, an abrupt increase in the slope at high $t$ region (fifth segment) was observed in $\mathrm{AOcOH}$ (Fig. 9e), indicating that macropores were present in these samples.

184 As for $\mathrm{APeOH}(600)$ and $\mathrm{AHeOH}(600)$, micropores were not recognized and the first and second segments are merged into one line going through the origin (Figs. 9c and 9d). This result can be explained by the boehmite layers separated by long alkyl groups. The forth and fifth segments are also merged. Usually the slope at high $t$ region (forth segment) corresponds to the outer surface area after mesopores are filled by adsorbate molecules by capillary condensation. However, this is not the case because the slope at

190 this region is much lager than the slope at low $t$ region (the first segment; corresponding to the total surface area). Therefore, pores are formed between the 192 irregularly-shaped particles (Fig. 4c) and the size of these pores distributes widely 193 from meso- to macro- pore region.

194 The most significant difference between the $t$ plots of $\mathrm{AHeOH}(1000)$ and

$195 \mathrm{ADDOH}(1000) \mathrm{A}$ is found in the slope at higher $t$ region (Fig. 9f). Since the slope for $196 \mathrm{ADDOH}(1000)$ is smaller than the slope at low $t$ region, the former corresponds to the outer surface area of the irregularly-shaped particles shown in Fig. 5d. The particles 
198 size was so large that the space between these particles is not recognized as pores by

199 the nitrogen adsorption method.

200 The pore sizes of the aluminas derived from ADDOH(as-syn) can be assessed either

201 by closure points of the hysteresis in the isotherms (Fig. 8a), by the regions of the third

202 segments in the $t$-plots (Fig. 9f) or by the pore-size distribution curves (Fig. 8b). All the

203 data suggest that the pore size increased with the increase in the calcination

204 temperature. However, the increase in the pore size by the increase in the calcination

205 temperature is much smaller than that for the ordinary aluminas derived from

206 pseudoboehmite and crystalline aluminum hydroxides [20]. This indicates robustness

207 of the pore structure of the alumina derived from the dodecyl derivative of boehmite,

208 which is attributed to the well-developed layer structure of the precursor due to the

209 strong interaction between the alkyl groups. Table 2 summarized the results for the

210 physical properties of the products.

\section{Conclusions}

The alumina derived from the ethyl derivative of boehmite had a broad pore-size

214 distribution, while the pore-size of the aluminas obtained from the alkyl derivatives of boehmite with long alkyl chains distributed in a narrow range in the mesopore region. 
216 The mode pore diameter of the latter aluminas increased with an increase in calcination

217 temperature (as-syn., $39 \AA \AA$; $600{ }^{\circ} \mathrm{C}, 54 \AA$; $800{ }^{\circ} \mathrm{C}, 58 \AA$; $1000{ }^{\circ} \mathrm{C}, 68 \AA \AA$; for ADDOH), 218 but narrow pore-size distribution was maintained even after calcination at high 219 temperatures. These results indicate that van der Waals interaction between the alkyl 220 chains facilitated the formation of the boehmite layers having a fewer number of 221 defects, and that the collapse of the well-developed boehmite layers gave aluminas 222 with narrow pore-size distributions.

\section{References}

225 [1] D.L. Trimm, A. Stanislaus, Appl. Catal. 21 (1986) 215.

226 [2] A.C. Pierre, E. Elaloui, G.M. Pajonk, Langmuir 14 (1998) 66.

227 [3] F. Vaudry, S. Khodabandeh, M.E. Davis, Chem. Mater. 8 (1996) 1451.

228 [4] S.A. Bagshaw, T.J. Pinnavaia, Angew. Chem. Int. Ed. Engl. 35 (1996) 1102.

229 [5] W. Zhang, T.J. Pinnavaia, Chem. Commun. (1998) 1185.

230 [6] S. Valange, J.-L. Guth, F. Kolenda, S. Lacombe, Z. Gabelica, Microporous Mesoporous Mater. 35-36 (2000) 597.

[7] X. Zhang, F. Zhang, K.-Y. Chan, Mater. Lett. 58 (2004) 2872.

233 [8] W.-C. Li, A.-H. Lu, W. Schmidt, F. Schüth, Chem. Eur. J. 11 (2005) 1658.

234 [9] A. Ionescu, A. Allouche, J.-P. Aycard, M. Rajzmann, F. Hutschka, J. Phys. Chem. B 
106 (2002) 9359.

236 [10] M. Inoue, H. Kominami, T. Inui, J. Am. Ceram. Soc. 73 (1990) 1100.

237 [11] M. Inoue, M. Kimura, T. Inui, Chem. Mater. 12 (2000) 55.

238 [12] M. Inoue, Y. Kondo, T. Inui, Inorg. Chem. 27 (1988) 215.

239 [13] M. Inoue, H. Tanino, Y. Kondo, T. Inui, Clays Clay Miner. 39 (1991) 151.

240 [14] J.J. Fripiat, H. Bosmans, P.G. Rouxhet, J. Phys. Chem. 71 (1967) 1097.

241

[15] M.C. Stegmann, D. Vivien, C. Mazieres, Spectrichim. Acta, Part A 29A (1973)

242 1653.

[16] A.B. Kiss, G. Keresztury, L. Farkas, Spectrochim. Acta. Part A 36A (1980) 653.

[17] Y. Yacoub, R. Bata, M. Gautam, Proc. Instn. Mech. Engrs. Part A 212 (1998) 363.

[18] J.H. de Boer, B.C. Lippens, J. Catal. 3 (1964) 38.

246

[19] B.C. Lippens, J.H. de Boer, J. Catal. 4 (1965) 319.

[20] T. Inui, T. Miyake, Y. Takegami, J. Jpn. Petrol. Inst. 25 (1983) 242. 


\section{Figure captions}

Fig. 1. XRD patterns of pseudoboehmite (h) and the products (a-g) obtained by reaction of AIP in alcohols: a, AEtOH(as-syn); b, ABuOH(as-syn); c, APeOH(as-syn); d, $\mathrm{AHeOH}($ as-syn); e, $\mathrm{AOcOH}($ as-syn); f, ADeOH(as-syn); g, ADDOH(as-syn).

Fig. 2. IR spectra of the products obtained by the reaction of AIP in alcohols: a,

AEtOH(as-syn); b, AHeOH(as-syn); c, ADDOH(as-syn).

Fig. 3. Thermal analyses of the products obtained by the reaction of AIP in the alcohols specified in the figure, in a $40 \mathrm{ml} / \mathrm{min}$ flow of dried air at the heating rate of $10{ }^{\circ} \mathrm{C}$ /min: a, TG; b, DTA.

Fig. 4. Scanning electron micrographs of the products: a, AEtOH(as-syn); b, APeOH(as-syn); c, AHeOH(as-syn); d, ADDOH(as-syn).

Fig. 5. XRD patterns of aluminas obtained by calcinaton of the hexyl derivative of boehmite at various temperature in air: a, $300{ }^{\circ} \mathrm{C}$; b, $400{ }^{\circ} \mathrm{C}$; c, $500{ }^{\circ} \mathrm{C}$; d, $600{ }^{\circ} \mathrm{C}$; e, $800{ }^{\circ} \mathrm{C}$; f, $1000{ }^{\circ} \mathrm{C} ; \mathrm{g}, 1100{ }^{\circ} \mathrm{C} ; \mathrm{h}, 1200{ }^{\circ} \mathrm{C}$.

274 calcinaton of the ethyl derivative of boehmite at various temperatures. 
277 Fig. 8. Nitrogen adsorption isotherms (a) and t-plots (b) of the aluminas obtained by 278 calcinaton of the dodecyl derivative of boehmite at various temperatures.

279 Fig. 9. $t$-Plots of the aluminas obtained by calcinaton of the alkyl derivatives of 280 boehmite at various temperatures: (a) ethyl derivative boehmite; (b) butyl derivative 281 boehmite; (c) pentyl derivative boehmite; (d) hexyl derivative boehmite; (e) octyl 282 derivative boehmite; (f) dodecyl derivative boehmite. 
Table 1

Summary of thermal analysis of the alkyl derivatives of boehmite obtained by solvothermal method.

\begin{tabular}{cccl}
\hline Sample & $\begin{array}{c}\text { Weight ratio } \\
\left(\mathrm{BD}^{\mathrm{a}} / \mathrm{Al}_{2} \mathrm{O}_{3}\right)\end{array}$ & $\begin{array}{c}\text { Ignition } \\
\text { temperature }\left({ }^{\circ} \mathrm{C}\right)\end{array}$ & Molecular fomular $^{\mathrm{b}}$ \\
\hline $\mathrm{AEtOH}$ & 1.25 & 425 & $\mathrm{AlO}(\mathrm{OH})_{0.86}(\mathrm{OEt})_{0.14}$ \\
$\mathrm{ABuOH}$ & 1.25 & 402 & $\mathrm{AlO}(\mathrm{OH})_{0.89}(\mathrm{OBu})_{0.11}$ \\
$\mathrm{APeOH}$ & 1.51 & 417 & $\mathrm{AlO}(\mathrm{OH})_{0.76}(\mathrm{OPe})_{0.24}$ \\
$\mathrm{AHeOH}$ & 1.36 & 407 & $\mathrm{AlO}(\mathrm{OH})_{0.89}(\mathrm{OHe})_{0.11}$ \\
$\mathrm{AOcOH}$ & 1.51 & 408 & $\mathrm{AlO}(\mathrm{OH})_{0.85}(\mathrm{OOC})_{0.15}$ \\
$\mathrm{ADeOH}$ & 1.41 & 400 & $\mathrm{AlO}(\mathrm{OH})_{0.91}(\mathrm{ODe})_{0.09}$ \\
$\mathrm{ADDOH}$ & 1.66 & 373 & $\mathrm{AlO}(\mathrm{OH})_{0.85}(\mathrm{ODD})_{0.15}$ \\
\hline
\end{tabular}

a: Alkyl derivatives of boehmite.

b: Empirical formulas, $\mathrm{AlO}(\mathrm{OH})_{1-x}(\mathrm{OR})_{x}$ were calculated from ignition losses determined by TG analysis for the products; Et, $\mathrm{CH}_{3} \mathrm{CH}_{2^{-}} ; \mathrm{Bu}, \mathrm{CH}_{3}\left(\mathrm{CH}_{2}\right)_{3^{-}} ; \mathrm{Pe}$, $\mathrm{CH}_{3}\left(\mathrm{CH}_{2}\right)_{4^{-}} ; \mathrm{He}, \mathrm{CH}_{3}\left(\mathrm{CH}_{2}\right)_{5^{-}}$; Oc, $\mathrm{CH}_{3}\left(\mathrm{CH}_{2}\right)_{7^{-}}$; De, $\mathrm{CH}_{3}\left(\mathrm{CH}_{2}\right)_{9^{-}} ; \mathrm{DD}, \mathrm{CH}_{3}\left(\mathrm{CH}_{2}\right)_{11^{-}}$ 
Table 2

Physical properties of the alumina obtained by calcination of alkyl derivatives of boehmite at various temperatures.

\begin{tabular}{|c|c|c|c|c|}
\hline Sample & $\begin{array}{l}\text { Calcination } \\
\text { Temp. }\left({ }^{\circ} \mathrm{C}\right)\end{array}$ & $\begin{array}{c}\text { Surface } \\
\text { area }\left(\mathrm{m}^{2} / \mathrm{g}\right)\end{array}$ & $\begin{array}{l}\text { Pore volume } \\
\text { (cc/g) }\end{array}$ & $\begin{array}{c}\text { mode pore } \\
\text { size }(\AA)\end{array}$ \\
\hline \multirow[t]{4}{*}{$\mathrm{AEtOH}$} & as syn. & 389 & 0.74 & 74 \\
\hline & 600 & 346 & 1.02 & 68 \\
\hline & 800 & 167 & 0.64 & 63 \\
\hline & 1000 & 117 & 0.64 & 80 \\
\hline \multirow[t]{4}{*}{$\mathrm{ABuOH}$} & as syn. & 543 & 0.63 & 37 \\
\hline & 400 & 414 & 0.73 & 50 \\
\hline & 600 & 390 & 0.73 & 51 \\
\hline & 1000 & 112 & 0.37 & 58 \\
\hline \multirow[t]{2}{*}{$\mathrm{APeOH}$} & 600 & 364 & 1.0 & 50 \\
\hline & 1000 & 105 & 0.56 & 110 \\
\hline \multirow[t]{3}{*}{$\mathrm{AHeOH}$} & as syn. & 389 & 0.47 & 90 \\
\hline & 600 & 392 & 0.69 & 68 \\
\hline & 1000 & 119 & 0.51 & 154 \\
\hline \multirow[t]{3}{*}{$\mathrm{AHOcOH}$} & 400 & 530 & 1.1 & 37 \\
\hline & 600 & 513 & 1.27 & 50 \\
\hline & 1000 & 136 & 0.65 & 62 \\
\hline \multirow[t]{4}{*}{$\mathrm{ADeOH}$} & as syn & 444 & 0.71 & 37 \\
\hline & 400 & 592 & 1.3 & 37 \\
\hline & 600 & 469 & 1.2 & 42 \\
\hline & 1000 & 157 & 0.76 & 62 \\
\hline \multirow[t]{4}{*}{$\mathrm{ADDOH}$} & as syn. & 392 & 0.54 & 39 \\
\hline & 600 & 338 & 0.69 & 54 \\
\hline & 800 & 258 & 0.59 & 58 \\
\hline & 1000 & 111 & 0.32 & 68 \\
\hline
\end{tabular}




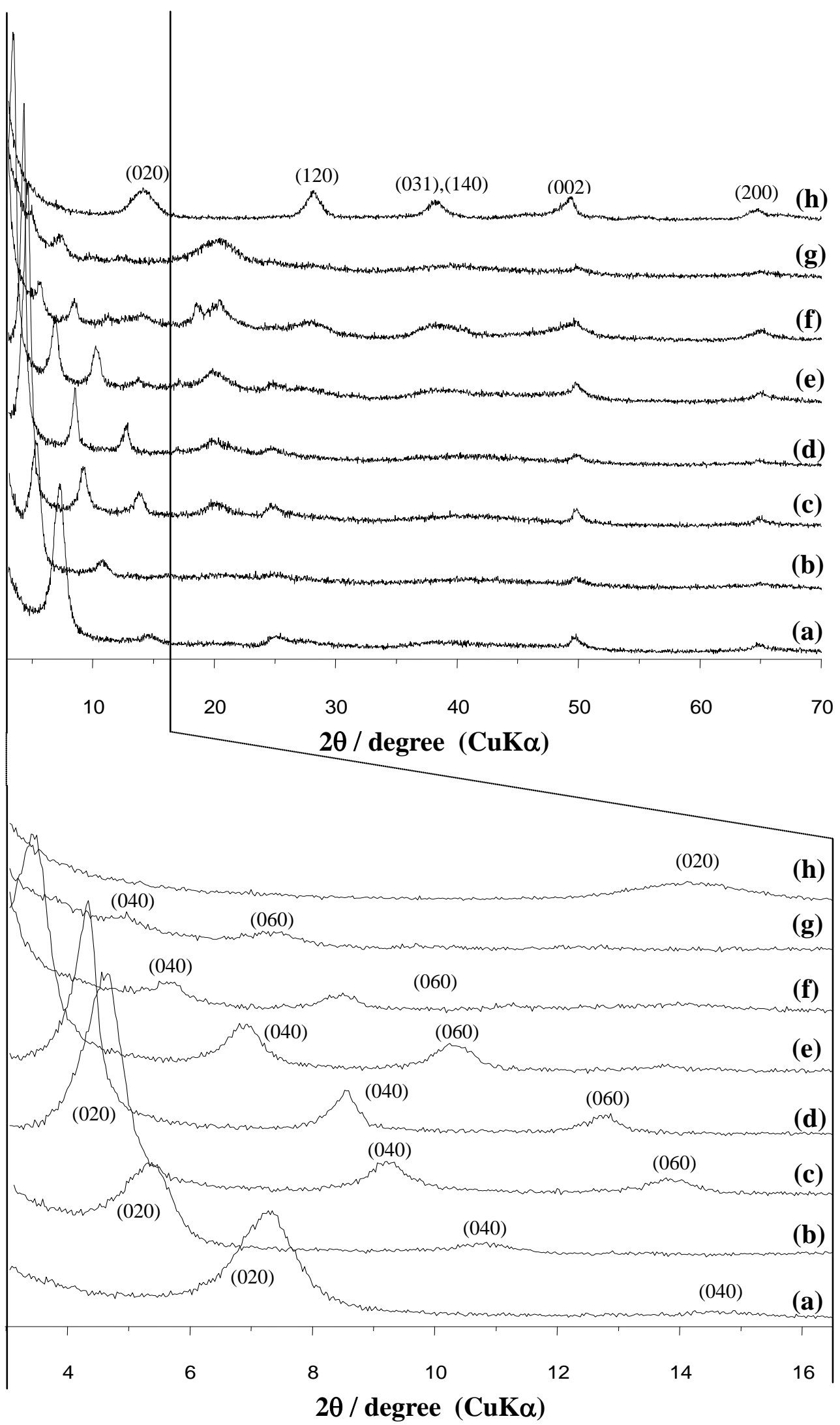

Fig. 1. Kim, et al. 


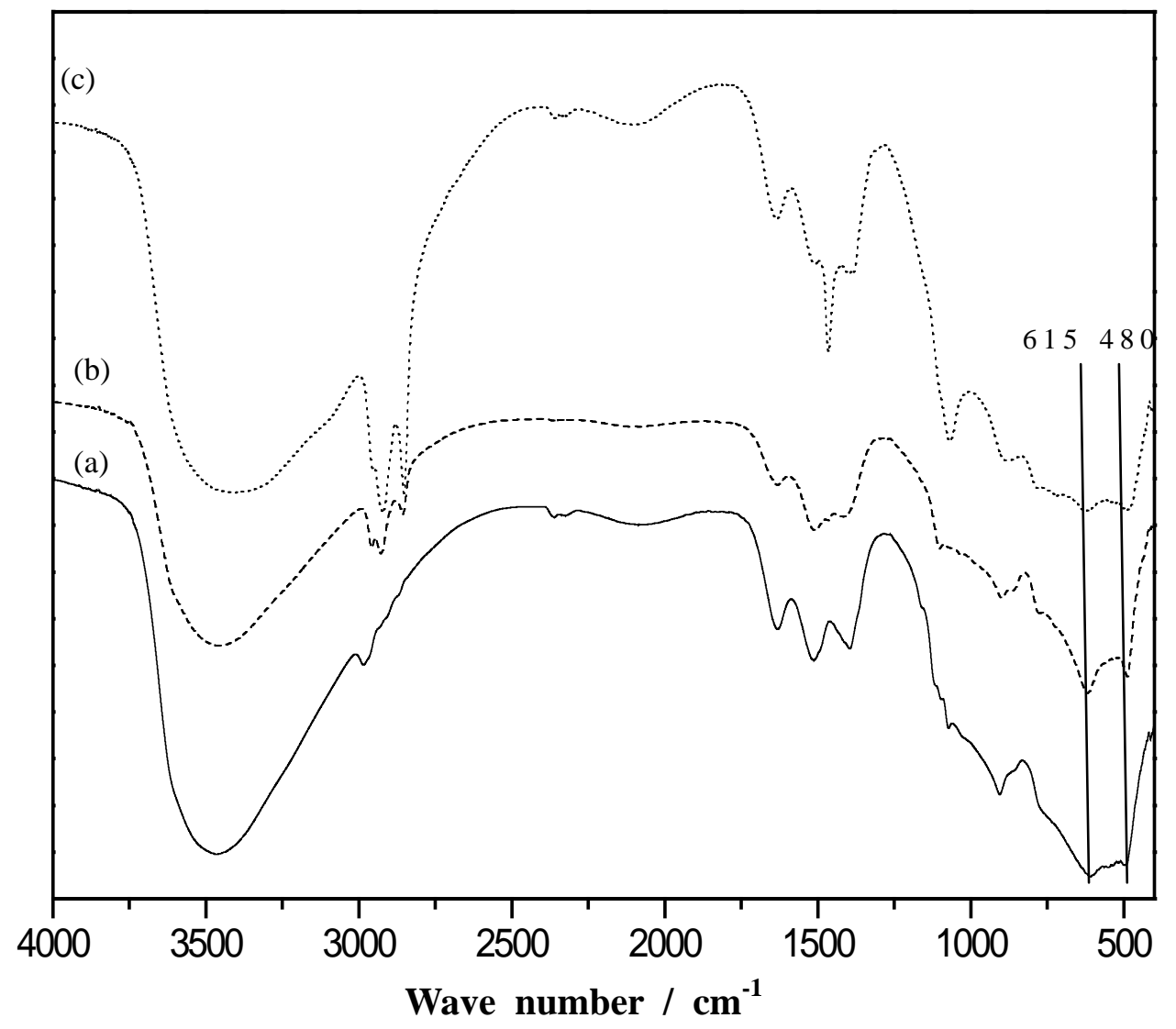

Fig. 2. Kim, et al. 

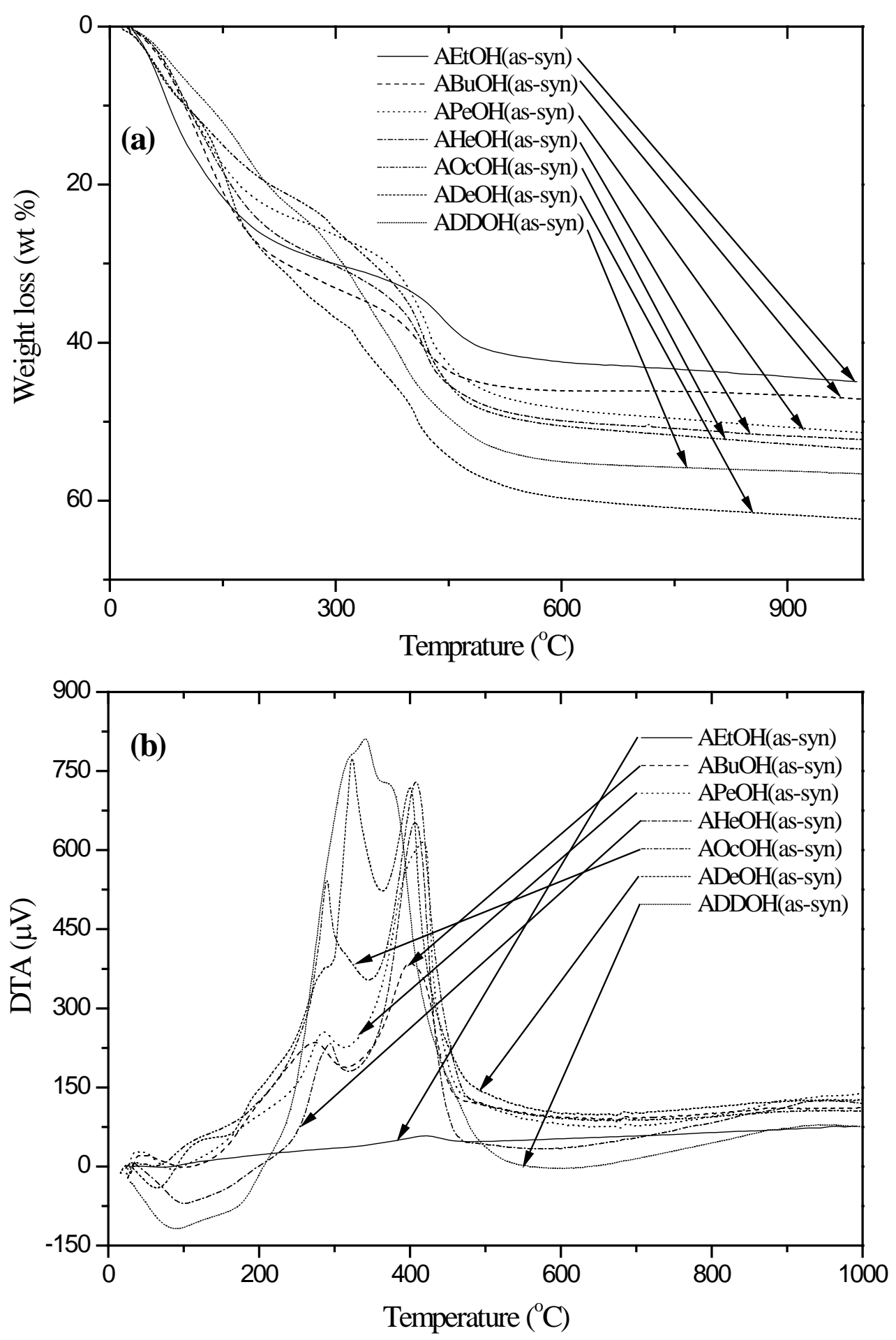

Fig. 3. Kim, et al. 

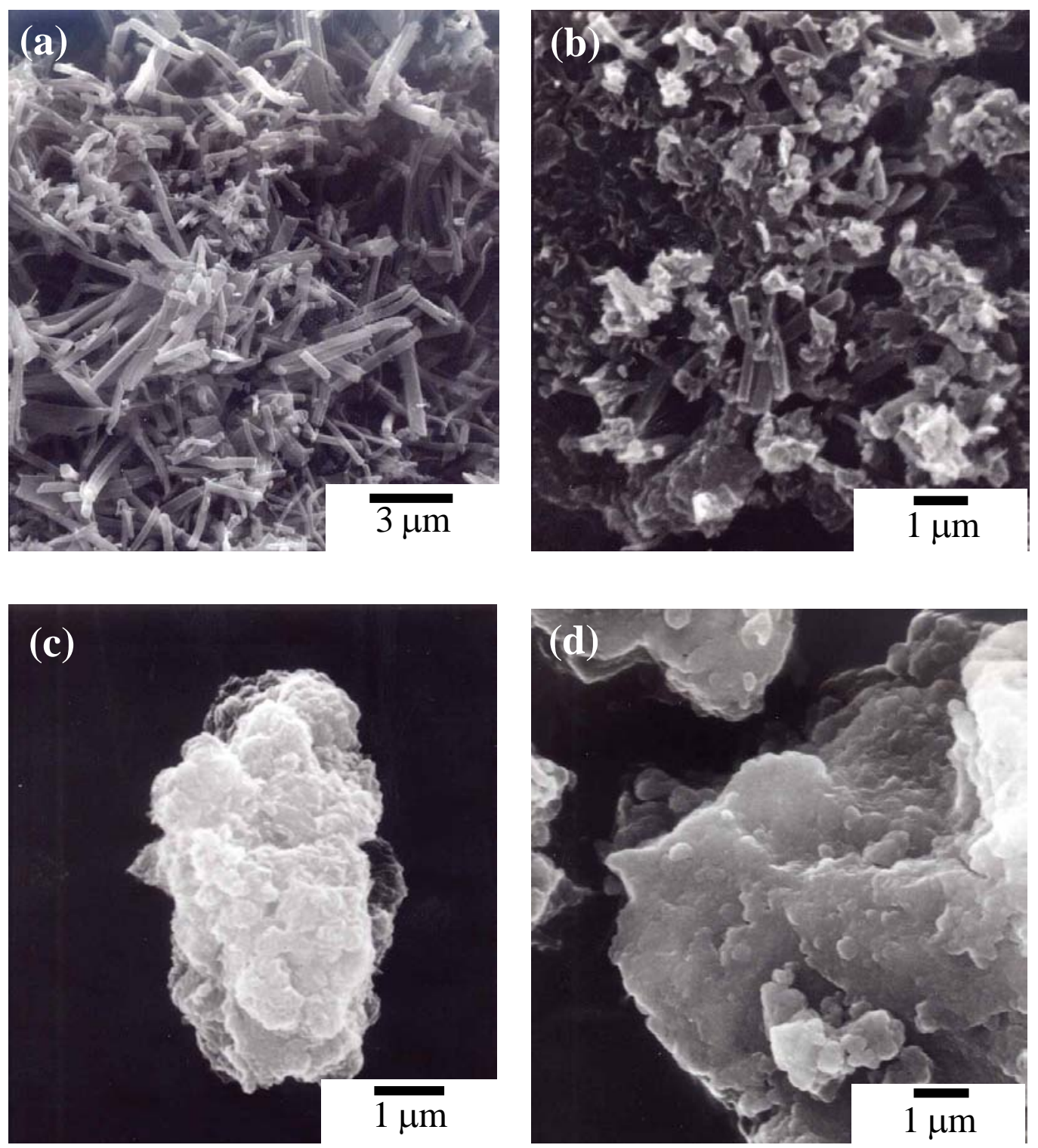

Fig. 4. Kim, et al. 


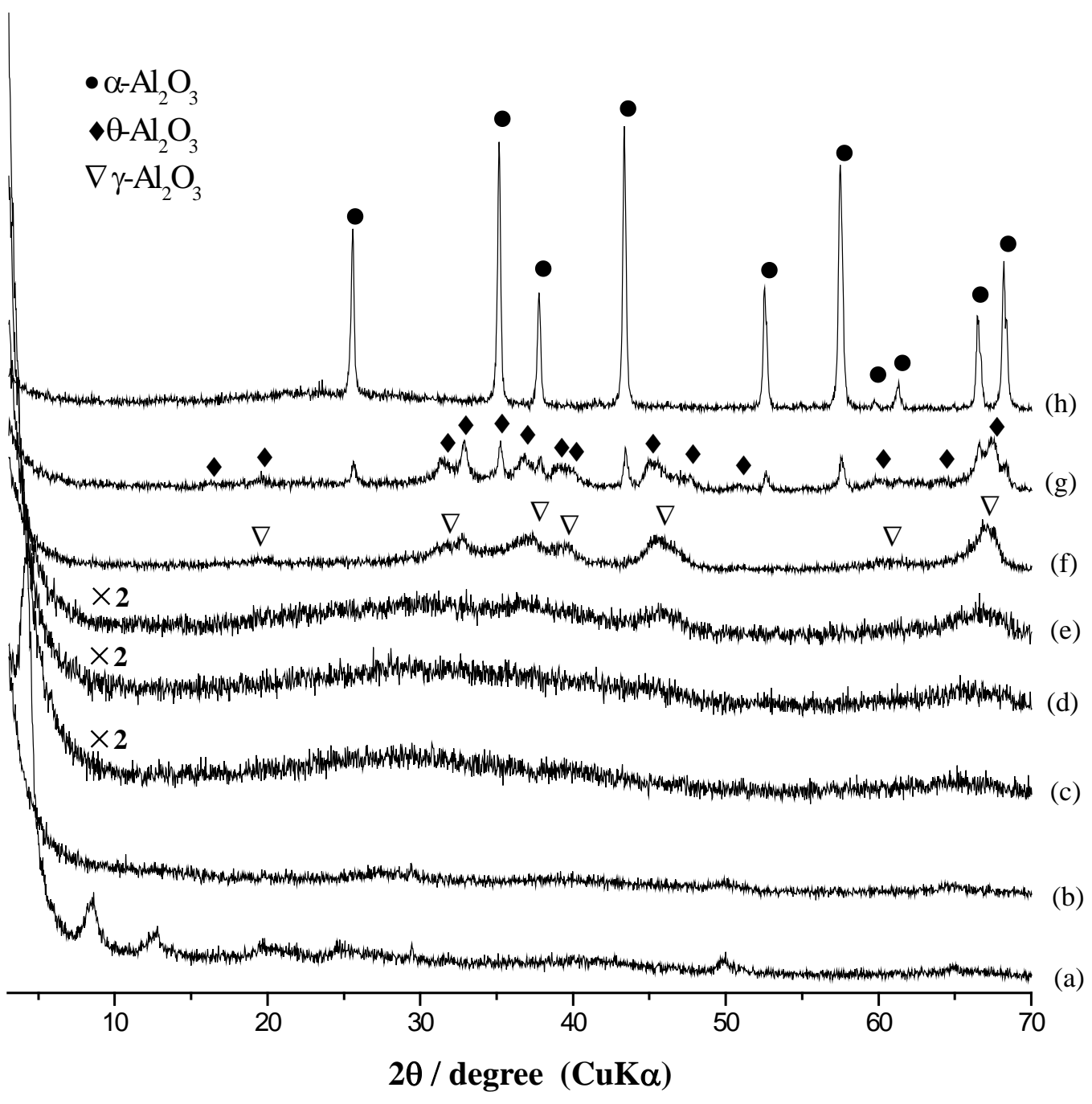

Fig. 5. Kim, et al. 

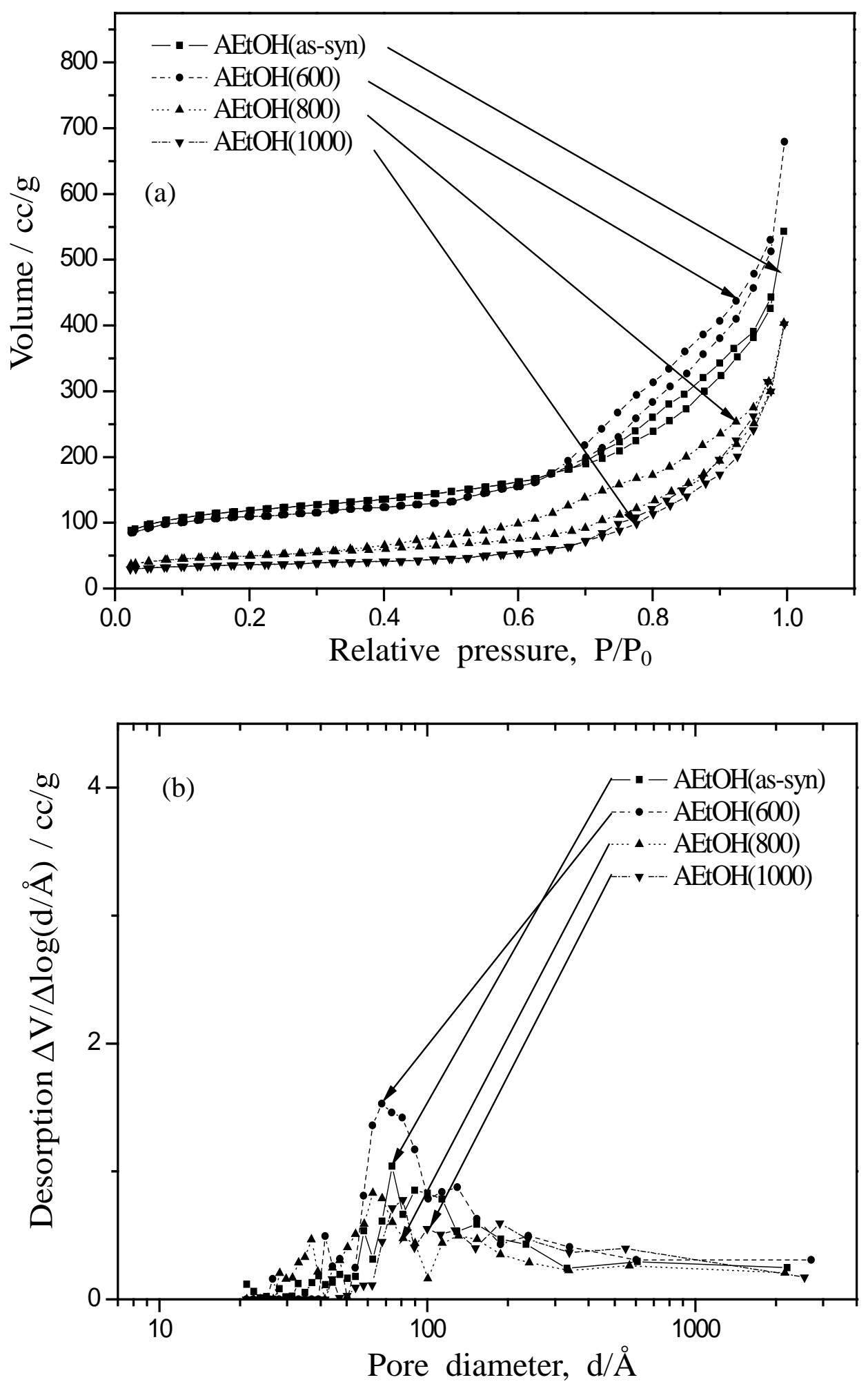

Fig. 6. Kim, et al. 

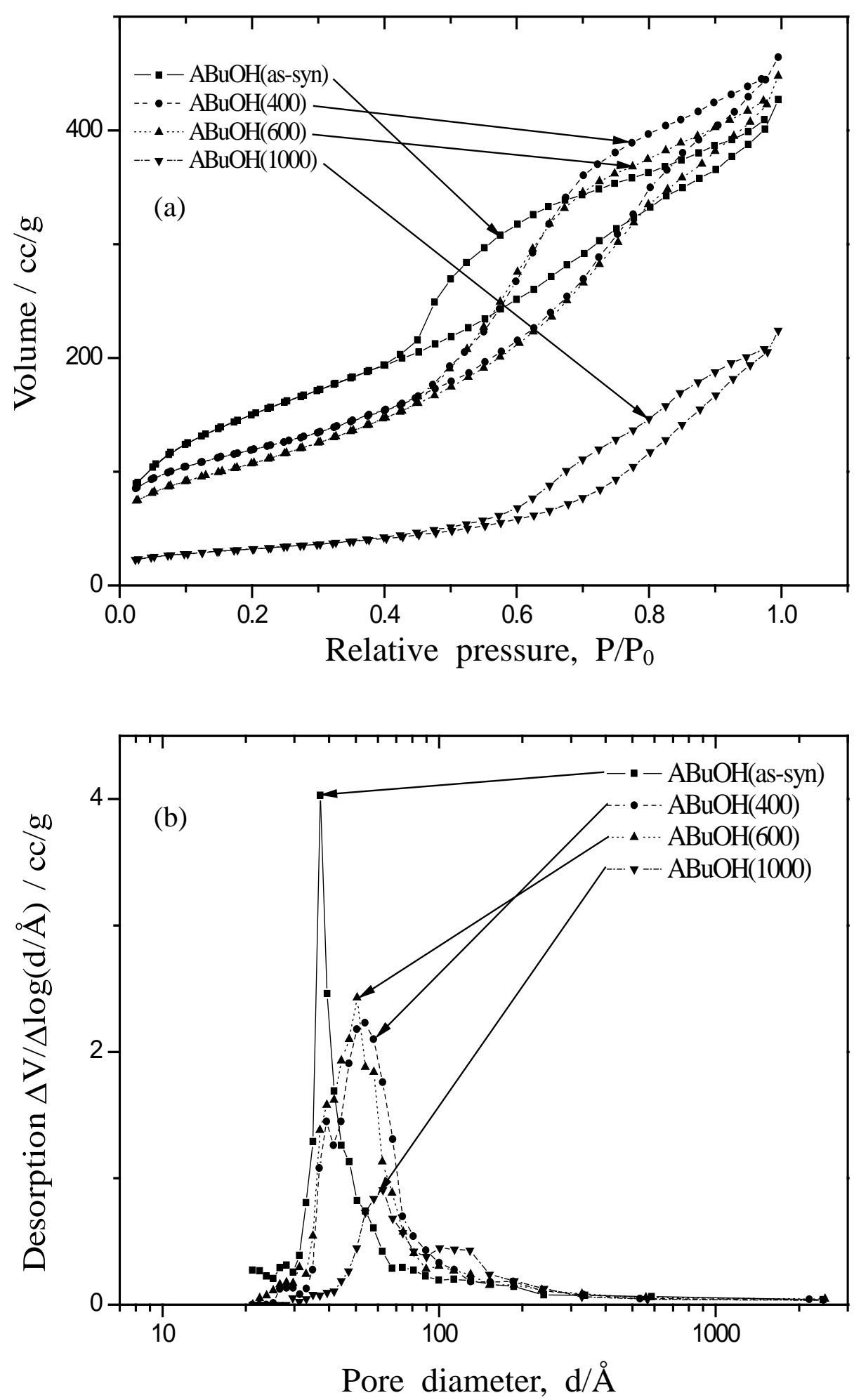

Fig. 7. Kim, et al. 

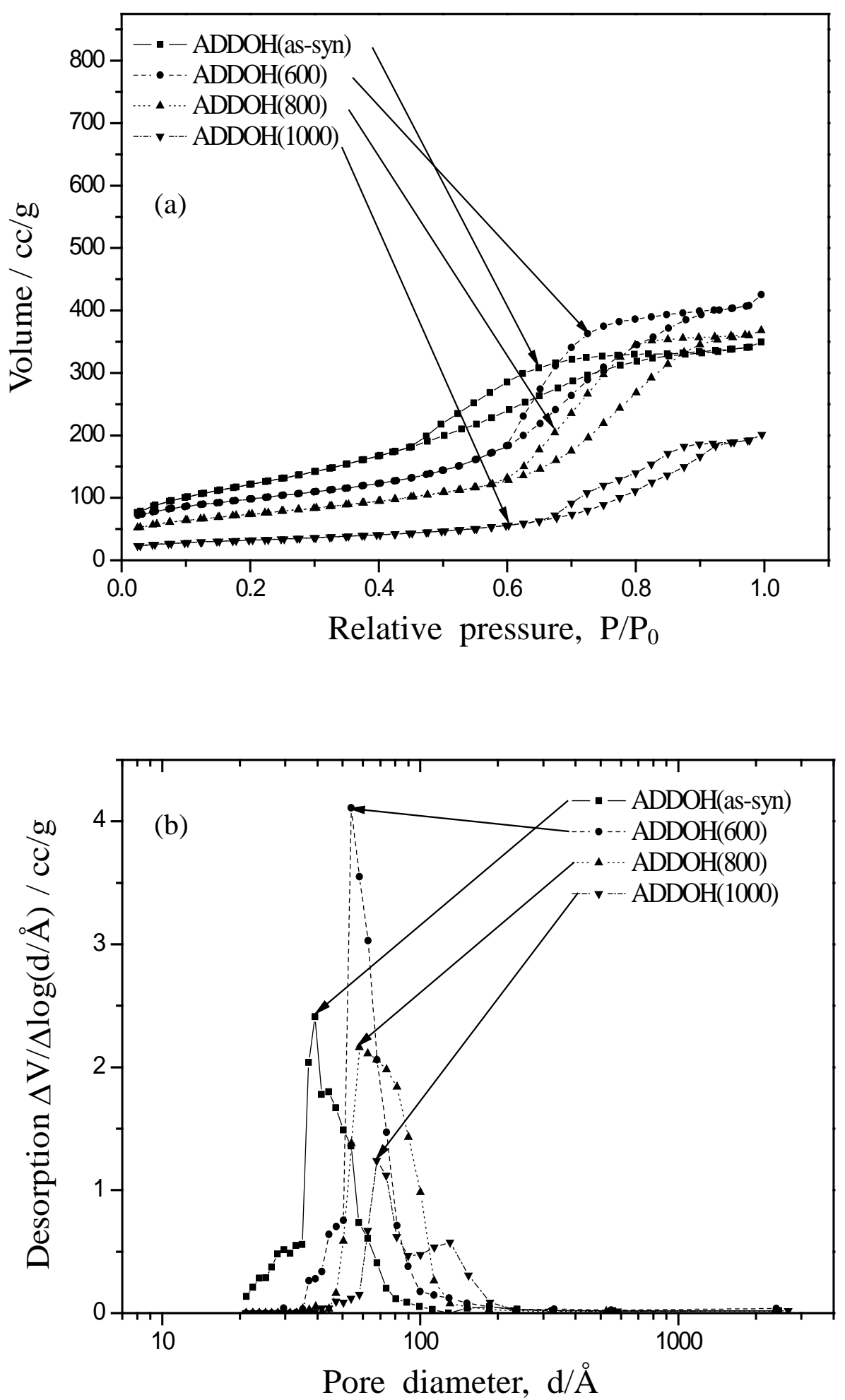

Fig. 8. Kim, et al. 

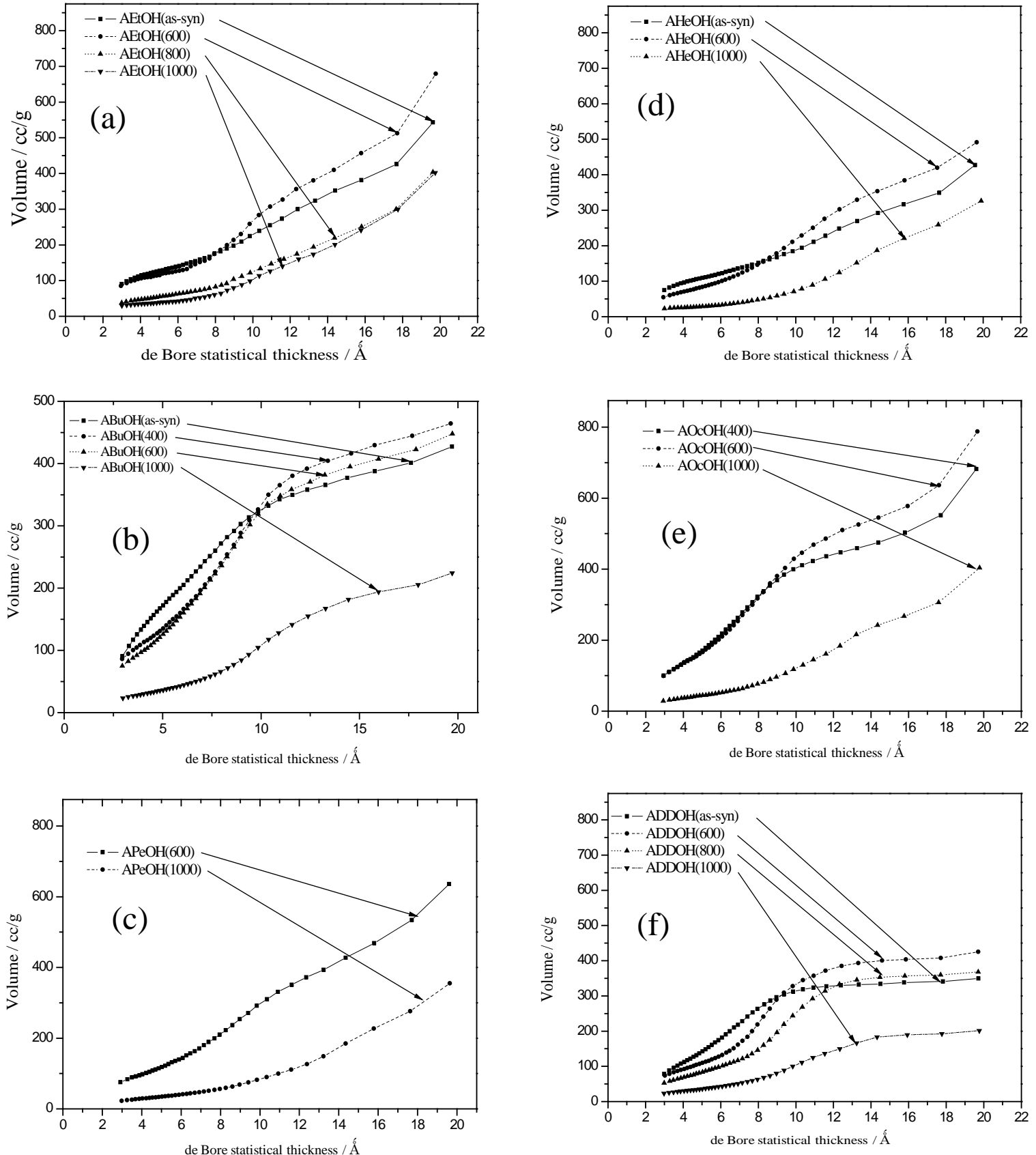

Fig. 9. Kim, et al. 